Irish Section Conference, 22-24 June 2021, Nutrition, health and ageing — translating science into practice - Part A

\title{
Diet and lifestyle behaviours in a sample of Irish higher education students
}

\author{
S. Doak ${ }^{1}$, J. Kearney ${ }^{2}$, J.M. McCormack ${ }^{1}$ and L. Keaver ${ }^{1}$ \\ ${ }^{1}$ Department of Health and Nutritional Science, Institute of Technology Sligo, Sligo, Ireland and \\ ${ }^{2}$ School of Biological and Health Sciences, Technological University Dublin, City Campus, Dublin, Ireland
}

The transition from secondary school into higher education may impact health-related behaviours due to a change of environment and living arrangements ${ }^{(1)}$. Many lifelong health-related behaviours are established at this time, that are likely to be maintained throughout life ${ }^{(2)}$. Evidence suggests that unhealthy diet and lifestyle behaviours may correlate and can cause an increase in co-morbidities, obesity and affect overall health ${ }^{(2)}$. The introduction of the Okanagan Charter, (2015) detailed the important role of health promotion within a higher education setting ${ }^{(3)}$. An improvement in the health of students may contribute to the improvement of the health and well-being of current and future communities and society at large ${ }^{(3)}$. The aim of this study is to assess the current dietary quality and lifestyle behaviours of students attending the Institute of Technology, Sligo (ITS) in the west of Ireland.

Full time students, aged 18+, were eligible to participate in this cross-sectional web-based survey, hosted by Qualtrics ${ }^{\circledR X M}$. Recruitment was conducted in November \& December 2020, through the class representative system, social media, and email, during varying stages of the COVID-19 pandemic. Self-reported information was collected in relation to demographics and smoking. A validated short-form food frequency questionnaire assessing dietary quality (scored from 5-15) was completed along with validated measures assessing alcohol use (Alcohol Use Disorders Identification Test Alcohol Consumption - AUDIT-C), sleep quality (Pittsburgh Sleep Quality Index - PSQI), perceived stress (Perceived Stress Scale-10 - PSS-10), and physical activity (International Physical Activity Questionnaire Short Form - IPAQ-SF). Statistical analysis was performed using chi-square, one-way ANOVA, and independent sample t-tests.

The cohort $(\mathrm{n}=238)$ were predominately female $(64 \%)$; mean age and body mass index (BMI) were $25.1 \pm 9.21$ and $24.75 \pm 5.10 \mathrm{~kg} / \mathrm{m} 2$, respectively, with $6 \%$ classified as underweight and $40 \%$ either overweight or obese. Students from all faculties and years within the institute engaged in the study; first year $(n=90,38 \%)$ and science students $(n=87,38 \%)$ were the most represented cohorts. Mean Dietary Quality Score was 10.02 \pm 1.86 . Mean fruit and vegetable consumption was $3.06 \pm 2.78$ a day and $79 \%(n=189)$ did not meet Irish RDA of five-a-day. Participants were predominately non-smokers $(n=175,73 \%)$, low risk alcohol consumers $(n=152$, $64 \%)$, had poor sleep quality $(\mathrm{n}=134,56 \%)$, were moderately stressed $(\mathrm{n}=165,69 \%)$, and moderately physically active $(\mathrm{n}=119$, $52 \%$ ). There was no statistically significant association found between diet quality score and BMI, smoking, alcohol use, sleep quality, stress, or physical activity.

This study provides data on the current dietary quality, lifestyle behaviours, and their associations in a sample of Irish higher education students. Results address a gap in the literature and will inform healthy campus initiatives in this area.

\section{References}

1. Crombie AP, Ilich JZ, Dutton GR, et al. (2009) Nutr Rev 67, 83-94

2. Gherasim A, Arhire LI, Nită O, et al. (2020) Proc Nutr Soc 79(3), 311-323

3. International Conference on Health Promoting Universities \& Colleges (2015) Okanagan Charter: An international charter for health promoting universities \& colleges 